Pacific Journal of Mathematics

A GEOMETRIC APPROACH TO THE FIXED POINT INDEX 


\title{
A GEOMETRIC APPROACH TO THE FIXED POINT INDEX
}

\author{
ROGER D. NUSSBAUM
}

\begin{abstract}
J. Leray defined a local fixed point index for functions defined in what he called convexoid spaces. From the standpoint of analysis, the most important example of a convexoid space is a compact subset $C \subset X, X$ a locally convex topological vector space, such that $C=\cup_{i=1}^{n} C_{i}$, where $C_{i}$ are compact, convex subsets of $X$ or a homeomorphic image of such a $C$. In this paper a simple geometric approach is given (see Lemma 2 below) by means of which a fixed point index can be defined for functions with domain in a class of spaces $\mathscr{F}$ which contains the spaces $C$ mentioned above and also the compact metric ANR's. The usual properties of the fixed point index are established, and it is shown that they axiomatically determine the index for the class of spaces $\mathscr{F}$.
\end{abstract}

As far as we know, none of the approaches to the fixed point index which have been published since Leray's work have been shown to apply to spaces $C$ of the type above. For instance, A. Granas [9] has remarked that if a compact space $C$ is $r$-dominated by an open subset of an lctvs, then the Leray-Schauder index for compact maps on open subsets of an lctvs gives a fixed point index for maps of open subsets of $C$ into $C$. But without metrizability the spaces considered here are not necessarily $r$-dominated by open subsets of an lctvs. F. Browder [3] has shown that if a compact Hausdorff space admits a "semicomplex structure," then a fixed point index can be defined for functions with domain in the space. However, to show that given topological spaces admit semicomplex structures, the metrizability of the spaces has almost invariable been used. Thus Browder has shown that compact, metric ANR's admit semicomplex structures, and Thompson [19] has established the same thing for metric HLC* spaces. But a finite union of compact, convex sets in an lctvs need not be metrizable. In any event, we shall avoid questions about semicomplex structure and obtain our fixed point index from the classical one for compact, finite dimensional polyhedra.

1. Let us begin with some notation. Let $C$ be a compact subset of a locally convex topological vector space (lctvs) $X$, always assumed Hausdorff. We shall write $C \in \mathscr{F}_{0}$ if there exists a finite closed covering $\left\{C_{i}: 1 \leqq i \leqq n\right\}$ of $C$ by compact, convex sets $C_{i} \subset C$, ie if $C=\bigcup_{i=1}^{n} C_{i}, C_{i}$ a compact convex subset of $X$. If $C \in \mathscr{F}_{0}, G \subset C$ 
is an open subset of $C$, and $f: G \rightarrow C$ is a continuous map such that $\{x \in G: f(x)=x\}$ is compact (possibly empty), then we shall define in this section a fixed point index $i_{G}(f, G)$ for $f$.

We take as a starting point Dold's development of the classical fixed point index [6]. If $Y$ is a compact Hausdorff space, Dold calls $Y$ a Euclidean neighborhood retract (ENR) if there exists an open subset 0 of some Euclidean space $\mathbf{R}^{n}$, a continuous map $i: Y \rightarrow 0$, and a continuous map $r: 0 \rightarrow Y$ such that $r \circ i=$ identity on $Y$. Notice that since any finite dimensional lctvs $F$ (always assumed Hausdorff) is linearly homeomorphic to $\mathbf{R}^{n}$ for some $n$, we may as well assume that $Y$ is imbedded in $F$ in the above definition of ENR. For our work here, the most important example of an ENR will be a compact subset $C$ of a finite dimensional lctvs $F$ such that $C=\bigcup_{i=1}^{n} C_{i}$ for some compact, convex subsets $C_{i} \subset F$. The fact that $C$ is an ENR follows from two theorems. First, Dugundji has shown [7] that a closed, convex subset of a Banach space $X$ is an ANR (see [1] for definitions). Since $F$ is finite dimensional it can be taken to be a Banach space, so any closed, convex subset of $F$ is an ENR. Second, a classical theorem states that if $Y_{1}$ and $Y_{2}$ are subsets of a metrizable space $Y$ and $Y_{1}, Y_{2}$, and $Y_{1} \cap Y_{2}$ are ANR's then $Y_{1} \cup Y_{2}$ is an ANR (see [1] for a proof). In our case these results combine to show $C$ is an ENR.

Now let $Y$ be a compact Hausdorff ENR, $G$ an open subset of $Y$, and $f: G \rightarrow Y$ a continuous map such that $\{x \in G: f(x)=x\}$ is compact. Then there is a unique integer valued function $i_{Y}(f, G)$ having the following properties. (O'Neill has shown uniqueness of the fixed point index for compact polytopes [17]. Since for any ENR $E$, there exists a polytope $P$, and continuous maps $j: E \rightarrow P, r: P \rightarrow E$ such that $r \circ j=$ identity on $E$, the methods of $\S 2$ show uniqueness for ENR's).

1. (The additivity property). Let $Y, f$, and $G$ be as above. If $S=\{x \in G: f(x)=x\}$, assume that $S \subset G_{1} \cup G_{2}$, where $G_{1}$ and $G_{2}$ are disjoint open subsets of $G$. Then $i_{Y}(f, G)=i_{Y}(f, G)+,i_{Y}\left(f, G_{2}\right)$. Further, if $i_{Y}(f, G) \neq 0, f$ has a fixed point in $G$.

2. (The homotopy property). Let $G$ be an open subset of a compact, Hausdorff ENR $Y$. Let $I=[0,1]=$ the closed unit interval and let $F: G \times I \rightarrow Y$ be a continuous map. Assume that $S=\{(x, t) \in G \times$ $I: F(x, t)=x\}$ is compact. Then if we define $F_{t}(x)=F(x, t), i_{Y}\left(F_{0}, G\right)=$ $i_{Y}\left(F_{1}, G\right)$.

3. (The normalization property). Let $Y$ be a compact, Hausdorff ENR and let $f: Y \rightarrow Y$ be a continuous map. Then $i_{Y}(f, Y)=\Lambda(f)$, 
where $\Lambda(f)$ is the Lefschetz number of $f$, using singular homology with rational coefficients.

4. (The commutativity property). Let $Y_{1}$ and $Y_{2}$ be compact, Hausdorff ENR's and let $G_{1}$ and $G_{2}$ be open subsets of $Y_{1}$ and $Y_{2}$ respectively. Let $f_{1}: G_{1} \rightarrow Y_{2}$ and $f_{2}: G_{2} \rightarrow Y_{1}$ be continuous maps. Setting $H_{1}=f_{1}^{-1}\left(G_{2}\right)$ and $H_{2}=f_{2}^{-1}\left(G_{1}\right)$, assume that $S_{1}=\left\{x \in H_{1}:\left(f_{2} f_{1}\right)(x)=x\right\}$ is compact. Then $S_{2}=\left\{x \in H_{2}:\left(f_{1} f_{2}\right)(x)=x\right\}$ is compact and $i_{Y_{1}}\left(f_{2} f_{1}, H_{1}\right)=$ $i_{Y_{2}}\left(f_{1} f_{2}, H_{2}\right)$.

Notice that if $Y, f$, and $G$ are as in 1 and $f(Y) \subset Y^{\prime} \subset Y, Y^{\prime}$ an ENR, then $i_{Y}(f, G)=i_{Y^{\prime}}\left(f, G \cap Y^{\prime}\right)$. This follows from 4 by using the inclusion $i: Y^{\prime} \rightarrow Y$.

In order to generalize the above fixed point index to our context, we need some lemmas. First, we introduce some further notation. We shall denote subsets of $\{1,2 \cdots, n\}$ by $J, K, L, M$ and we define $|J|$ to be the number of elements in $J$. If $C$ is a compact Hausdorff space, and $C=\bigcup_{i=1}^{n} C_{i}$, where $C_{i}$ is a compact subset of $C$ for $1 \leqq i \leqq n$, then for $L \subset\{1,2 \cdots, n\}$, we shall write $C_{L}=\bigcap_{i \in L} C_{i}$.

With the aid of this notation we can state our first lemma, which is the basis of all our further work.

LEMMA 1. Let $C$ be a compact, Hausdorff space such that $C=$ $\bigcup_{i=1}^{n} C_{i}, C_{i}$ a compact subset of $C$. Let $\mathscr{Q}$ be a finite open covering of $C$. Then there exists a finite open covering $\mathscr{V}=\left\{V_{J, i}: J \subset\{1,2 \cdots, n\}\right.$, $\left.1 \leqq i \leqq k_{J}\right\}$ (i.e., indexed by ordered paris $(J, i), J$ a subset of $\{1,2, \cdots, n\}$, i an integer for which $1 \leqq i \leqq k_{J}, k_{J}$ an integer depending on $J$ ) such that (1) $\mathscr{V}$ is a refinement of $\mathscr{U}$ (2) $V_{J, i}$ is empty if $C_{J}$ is empty and $V_{J, i} \cap C_{J}$ is nonempty if $V_{J, i}$ is nonempty. (3) If $k \notin J, \operatorname{cl}\left(V_{J, i}\right) \cap$ $C_{k}$ is empty. (4) If $|L| \geqq|K|$ but $L \not \supset K, V_{L, i} \cap V_{K, j}$ is empty.

Proof. We construct $\left\{V_{J, i}\right\}$ by induction on $|J|$, starting with $|J|=n$. The inductive assumption at the $(n-r)^{\text {th }}$ step, $1<r \leqq n$, is that there exists a collection of open sets

$$
\left\{V_{J, i}: J \subset\{1,2, \cdots, n\},|J| \geqq r, 1 \leqq i \leqq k_{J}\right\}
$$

which satisfies $1-4$ above and is such that $\bigcup_{|J| \geqq r} \bigcup_{i=1}^{k_{J}} V_{J, i} \supset \bigcup_{|J| \geqq r} C_{J}$. The object is to define open sets $V_{J, i}$ for $J \subset\{1,2, \cdots, n\}$ for which $|J|=r-1$ and $1 \leqq i \leqq k_{J}$ and such that $\left\{V_{J, i}:|J| \geqq r-1,1 \leqq i \leqq k_{J}\right\}$ satisfies 1-4 and gives an open covering of $U_{|J| \geqq r-1} C_{J}$.

Step 1. If $|J|=n$, let $\left\{V_{J, i}: 1 \leqq i \leqq k_{J}\right\}$ be the collection of $U \in \mathscr{Q}$ which have nonempty intersection with $C_{J}$. This collection may be empty. 
Step 2. Assume for some $r, 1 \leqq r \leqq n$, we have constructed $\left\{V_{J, i}:|J| \geqq r, 1 \leqq i \leqq k_{J}\right\}$ which satisfies the inductive hypothesis. If $r=1$, we are done, so assume $r>1$. For each $K \subset\{1,2, \cdots, n\}$ with $|K|=r-1$, let $A_{K}=C_{K}-\bigcup_{|L| \geqq r} \bigcup_{1 \leqq i \leqq k_{L}} V_{L, i}$ and notice that if $j \notin$ $K, A_{K}$ and $C_{j}$ are disjoint compact sets (since, for $j \notin K, C_{K} \cap C_{j}=$ $C_{K \cup\{j\}}$ and $\left.|K \cup\{j\}| \geqq r\right)$. It is also clear that $A_{K} \cap A_{K^{\prime}}$ is empty for all $K^{\prime}$ such that $K^{\prime} \neq K$, since $A_{K^{\prime}} \subset C_{j}$ for $j \in K^{\prime}-K$ and $A_{K} \cap C_{j}$ is empty. It is thus not hard to see that there exist open neighborhoods $O_{K}$ of $A_{K}$ for all $K \subset\{1,2, \cdots, n\}$ with $|K|=r-1$ such that $\operatorname{cl}\left(O_{K}\right) \cap C_{j}$ is empty for $j \notin K$ and $\operatorname{cl}\left(O_{K}\right) \cap \operatorname{cl}\left(O_{K^{\prime}}\right)$ is empty for all $K$, $K^{\prime}$ with $|K|=\left|K^{\prime}\right|=r-1$ but $K \neq K^{\prime}$.

Next, for a given $K \subset\{1,2, \cdots, n\}$ with $|K|=r-1$, consider all $L \subset\{1,2, \cdots, n\}$ such that $|L|>r-1$ but $L \supset K$. For each such $L$, select $j \in K$ such that $j \notin L$. If $(L, j)$ is such a pair, we know by inductive assumption that $\mathrm{cl}\left(\bigcup_{i=1}^{k L} V_{L, i}\right) \cap C_{j}$ is empty so there exists an open neighborhood $W_{(L, j)}$ of $C_{j} \supset C_{K}$ such that $\operatorname{cl}\left(\bigcup_{i=1}^{k} V_{K, i}\right) \cap W_{(L, j)}$ is empty. We set $W_{K}=\bigcap_{(L, j)} W_{(L, j)}$, where the intersection is taken over all ordered pairs $(L, j)$ as above. We define $T_{K}=O_{K} \cap W_{K}$, an open neighborhood of $A_{K}$.

Let $\left\{V_{K, i}: 1 \leqq i \leqq k_{K}\right\}$ be the collection of sets of the form $T_{K} \cap$ $U, U \in \mathscr{U}$, such that $A_{K} \cap U$ is nonempty. This collection may be empty. Clearly $\left\{V_{K, i}: 1 \leqq i \leqq k_{K}\right\}$ is an open covering of $A_{K}$. Observe also that $\left\{V_{J, i}:|J| \geqq r-1,1 \leqq i \leqq k_{J}\right\}$ gives an open covering of $U_{|J| \geqq r-1} C_{J}$. By induction we certainly have an open covering of $U_{|J| \geqq r} C_{J}$. If $x \in C_{K}$ for $|K|=r-1$ and $x \notin U_{|L| \geq r} V_{L, i}$, then by definition $x \in A_{K}$ so that $x \in V_{K, i}$ for some $i$.

It remains to check conditions 1-4 for the new covering. Since $V_{K: i},|K|=r-1$, was selected so $V_{K i} \subset U$ for some $U \in \mathscr{C}$, condition 1 holds. Condition 2 holds trivially: If $C_{K}$ is empty, $A_{K}$ is empty and $\left\{V_{K, i}: 1 \leqq i \leqq k_{K}\right\}$ is empty. If $V_{K} i$ is nonempty, $V_{K i}=U \cap T_{K}$ for some $U \in \mathscr{Q}$ such that $U \cap A_{K}$ is nonempty. If $|L| \geqq|K|$ but $L \supset K$, we want to show $V_{L, i} \cap V_{K \cdot j}$ is empty. If $|K|>r-1$, the result is true by inductive hypothesis. If $|K|=r-1$, and $|L|>r-1$, select $k \in K$ such that $k \notin L$. By our construction we have $V_{K \cdot j} \subset W_{K} \subset$ $W_{(L, k)}$ and $W_{(L k)} \cap \operatorname{cl}\left(U_{t=1}^{k} V_{L, t}\right)$ is empty, so that in particular $V_{L, i} \cap$ $V_{K, j}$ is empty. If $|K|=|L|=r-1$, then again by our construction, $V_{K, j} \cap V_{L: i} \subset O_{K} \cap O_{L}$ is empty. In either event, 4 is satisfied. Finally, to show 3 it suffices to show that if $|K|=r-1$ and $j \notin K, \operatorname{cl}\left(V_{K} i\right) \cap C_{j}$ is empty. Since $V_{K i} \subset O_{K}$, and $\operatorname{cl}\left(O_{K}\right) \cap C_{j}$ is empty, this follows immediately from our construction.

This completes the inductive step. After $n$ repetitions, the desired covering is obtained.

Our next lemma provides the justification for proving Lemma 1. 
Lemma 2 is the basic result upon which all work in this section depends.

Lemma 2. Let $C$ be a compact subset of an lctvs $X$ and suppose $C=U_{i=1}^{n} C_{i}, C_{i}$ a compact, convex subset of $X$. Then given any continuous seminorm $q$ and $\varepsilon>0$, there exists a continuous, finite dimensional map $r: C \rightarrow C$ such that if $x \in C_{i}$ for $1 \leqq i \leqq n$, then $r(x) \in C_{i}$ also and $q(r(x)-x) \leqq \varepsilon$. (By finite dimensional we mean that the range of $r$ lies in some finite dimensional subspace of $X$ ).

Proof. For each $x \in C$, let $U_{x}=\{y \in C: q(y-x)<\varepsilon / 2\}$. This gives an open covering of $C$, and by the compactness of $C$, there exists a finite subcovering $\mathscr{U}=\left\{U_{x_{1}}, U_{x_{2}}, \cdots, U_{x_{t}}\right\}$. Clearly, if $U \in \mathscr{U}$ and $x, y \in$ $U$, then $q(x-y)<\varepsilon$. By Lemma 1 , there exists a refinement $\mathscr{V}=$ $\left\{V_{J, i}: J \subset\{1,2, \cdots, n\}, 1 \leqq i \leqq k_{J}\right\}$ satisfying conditions $1-4$ of Lemma 1. By condition 2, for each nonempty $V_{J, i}$ select $P_{J, i} \in V_{J, i} \cap C_{J}$; also define $P_{J, i}=0$ if $V_{J, i}$ is empty. Let $\left\{\phi_{J, i}: J \subset\{1,2, \cdots, n\}, 1 \leqq i \leqq k_{J}\right\}$ be a partition of unity subordinate to $V_{J, i}$ (with the convention that $\phi_{J, i} \equiv 0$ if $V_{J, i}$ is empty), so that supp $\left(\phi_{J, i}\right) \subset V_{J, i}$ and $\sum_{J, i} \phi_{J, i}(x)=1$ for $x \in C$. Define $r(x)=\sum_{J, i} \phi_{J, i}(x) P_{J, i}$. It is clear that $r$ is a continuous, finite dimensional map. Also, it is easy to show that $q(x-r(x)) \leqq$

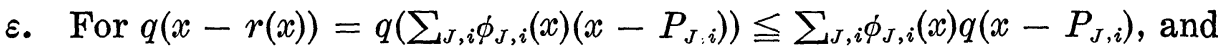
if $\phi_{J, i}(x) \neq 0, x \in V_{J, i}$, so that $q\left(x-P_{J, i}\right) \leqq \varepsilon$ and $\sum_{J, i} \phi_{J, i}(x) q\left(x-P_{J, i}\right) \leqq$ $\sum_{J, i} \phi_{J, i}(x) \varepsilon=\varepsilon$.

The nontrivial statement (and the reason for introducing $\left\{V_{J, i}\right\}$ ) is that $r(x) \in C_{i}$ if $x \in C_{i}$. Thus suppose $x \in C_{i}$. If for $L \subset\{1,2, \cdots, n\}$, $i \notin L$, we know by property 3 of the covering that $\operatorname{cl}\left(V_{L, j}\right) \cap C_{i}$ is empty, so that $\phi_{L, j}(x)=0$ (since $\left.\operatorname{supp} \phi_{L, j} \subset V_{L, j}\right)$. Thus we have $r(x)=$ $\sum_{(L, j), i \in L} \phi_{L, j}(x) P_{L, j}$. Since $P_{L, j} \in C_{L} \subset C_{i}$, this is just a convex combination of points in $C_{i}$ and hence lies in $C_{i}$.

Notice that the proof of Lemma 2 only uses properties 1-3 of the covering $\left\{V_{J, i}\right\}$.

Before proceeding with our main line of development, let us state a proposition which indicates again the usefulness of Lemma 1. The following proposition is standard if $C$ is a compact metric ANR, and it plays a key role in some developments of the fixed point index for compact, metric ANR's. Since we shall not need this result, we shall not give a proof except to say that it follows straightforwardly from Lemma 1. We refer the reader to Hanner's article [10], where theorems along the the general lines of the following proposition are proved for metric ANR's.

Proposition. Let $C$ be a compact subset of an lctvs $X$ and suppose 
$C=\bigcup_{i=1}^{n} C_{i}, C_{i}$ compact, convex. Let $\alpha$ be a finite open covering of $C$. Then there exists a finite open covering $\beta$ of $C$ which is refinement of $\alpha$ and has the following property: If $N_{\beta}$ denotes the nerve of $\beta$, then there exist continuous maps $f_{\beta}: C \rightarrow N_{\beta}$ and $g_{\beta}: N_{\beta} \rightarrow C$ such that $g_{\beta}$ of $f_{\beta}$ is homotopic to the identity $I$ on $C$. Furthermore the homotopy $F_{t}: C \rightarrow C, 0 \leqq t \leqq 1, F_{0}=g_{\beta} \circ f_{\beta}, F_{1}=I$, can be chosen so that for each $x \in C, \exists U \in \alpha$ for which $F_{t}(x) \in U$ for $0 \leqq t \leqq 1$.

With the aid of Lemma 2 we can define our fixed point index. Let $C$ be a compact subset of an lctvs $X$ and $G$ an open subset of $C$. Suppose that $C=\bigcup_{i=1}^{m} C_{i}, C_{i}$ compact and convex. Let $f: \operatorname{cl}(G) \rightarrow C$ be a continuous map such that $f(x) \neq x$ for $x \in \partial G=\mathrm{cl}(G)-G$. Since $\{x-f(x): x \in \partial G\}$ is a compact set not containing 0 and $X$ is an lctvs, there exists a continuous seminorm $q$ such that $q(f(x)-x) \geqq \varepsilon>0$ for $x \in \partial G$. We shall say that a continuous map g: $\mathrm{cl}(G) \rightarrow C$ is an admissible approximation with respect to $\left\langle f,\left\{C_{i}\right\}\right\rangle\left(C_{i}\right.$ as above) if (1) There exists a continuous seminorm $q$ such that $q(f(x)-x) \geqq \varepsilon>0$ for $x \in \partial G$ and $q(f(x)-g(x))<\varepsilon$ for $x \in \partial G$. (2) For all $x \in \operatorname{cl}(G)$, if $f(x) \in C_{i}$, then $g(x) \in C_{i}$ (3) $g$ is finite dimensional map, i.e. the range of $g$ lies in a finite dimensional subspace of $X$. With the notation above we take $C_{i}^{\prime}, 1 \leqq i \leqq m$, to be any finite dimensional compact, convex subset of $C_{i}$ such that $g(G) \subset \bigcup_{i=1}^{m} C_{i}^{\prime} \equiv C^{\prime}$. (If $g$ is as above and $V$ is any finite dimensional vector space containing the range of $g$, we can define $\left.C_{i}^{\prime}=C_{i} \cap V\right)$. By our previous remarks $C^{\prime}$ is a compact ENR. Notice that $g: \mathrm{cl}(G) \rightarrow C^{\prime}$, and by condition 1 on $g, g(x) \neq x$ for $x \in \partial G$. Thus we see that $i_{C^{\prime}}\left(g, G \cap C^{\prime}\right)$ is defined. We shall show below that if we define $i_{C}(f, G)=i_{C^{\prime}}\left(g, G \cap C^{\prime}\right)$, this gives a well defined definition.

THEOREM 1. Let $C$ be a compact subset of an letvs $X$ and assume $C=\bigcup_{i=1}^{t} E_{i}, E_{i}$ a compact, convex set. Let $G$ be an open subset of $C$ and $f: \mathrm{cl}(G) \rightarrow C$ a continuous may such that $f(x) \neq x$ for $x \in \partial G$. Let $q$ be any continouous seminorm and $\varepsilon>0$. Then there exists an admissible approximation $\theta$ with respect to $<f,\left\{E_{i}\right\}>$ such that $q(\theta(x)-$ $f(x))<\varepsilon$ for all $x \in \operatorname{cl}(G)$. Furthermore, suppose $C=\bigcup_{i=1}^{m} C_{i}$ and $C=\bigcup_{j=1}^{n} D_{j}, C_{i}$ and $D_{j}$ compact and convex. If $g$ is an admissible approximation with respect to $\left\langle f,\left\{C_{i}\right\}\right\rangle, h$ is an admissible approximation with respect to $\left\langle f,\left\{D_{j}\right\}\right\rangle, C_{i}^{\prime}, 1 \leqq i \leqq m$, is a finite dimensional compact, convex subset of $C_{i}$ such that $g(G) \subset \bigcup_{i=1}^{m} C_{i}^{\prime} \equiv C^{\prime}$ and $D_{j}^{\prime}, 1 \leqq j \leqq n$, is a finite dimensional compact, convex subset of $D_{j}$ such that $h(G) \subset \bigcup_{j=1}^{n} D_{j}^{\prime} \equiv D^{\prime}$, then $i_{C^{\prime}}\left(g, G \cap C^{\prime}\right)=i_{D^{\prime}}\left(h, G \cap D^{\prime}\right)$.

Proof. Let $q$ be as above and let $p$ be a continuous seminorm such that $p(f(x)-x) \geqq \delta>0$ for $x \in \partial G$. Define $q^{\prime}(x)=\max \{p(x), q\{(x)\}$ 
and $\varepsilon^{\prime}=\min \{\varepsilon, \delta\}$; of course $q^{\prime}$ is a continuous seminorm. By Lemma 2 , there exists a continuous map $r: C \rightarrow C$ such that

(1) $q^{\prime}(r(x)-x)<\varepsilon^{\prime}$ for all $x \in C$

(2) $r(x) \in E_{i}$ if $x \in E_{i}$ for all $x \in C$ and $1 \leqq i \leqq t$.

(3) $r$ is a finite dimensional map.

We define $\theta(x)=r(f(x))$. It is immediate that $\theta$ is an admissible approximation with respect to $<f,\left\{E_{i}\right\}>$.

Now let $g$ be an admissible approximation with respect to $\left\langle f,\left\{C_{i}\right\}\right\rangle$ and $h$ an admissible approximation with respect to $\left\langle f,\left\{D_{j}\right\}\right\rangle$. Thus there exist continuous seminorms $q_{1}$ and $q_{2}$ such that $q_{1}(f(x)-x) \leqq \varepsilon_{1}$, $q_{1}(f(x)-g(x))<\varepsilon_{1}, q_{2}(f(x)-x) \geqq \varepsilon_{2}$, and $q_{2}(f(x)-g(x))<\varepsilon_{2}$ for all $x \in \partial G$. We define $\widetilde{q}(x)=\max \left\{q_{1}(x), q_{2}(x)\right\}$, a continuous seminorm, and $\varepsilon=\min \left\{\varepsilon_{1}, \varepsilon_{2}\right\}$. Notice that $C=\bigcup_{i=1}^{m} \bigcup_{j=1}^{n} C_{i} \cap D_{j}, C_{i} \cap D_{j}$ a compact, convex subset of $X$. If we set $E_{i, j}=C_{i} \cap D_{j}$, by the first part of this theorem, there exists an admissible approximation $\theta$ with respect to $<f,\left\{E_{i, j}\right\}>$ such that $\widetilde{q}(f(x)-\theta(x))<\varepsilon$ for $x \in \operatorname{cl}(G)$.

Before proceeding further, let us recall the elementary theorem that if $A$ and $B$ are compact, convex subsets of a topological vector space $X$, then $\{s x+(1-s) y: 0 \leqq s \leqq 1, x \in A, y \in B\}$ is a compact, convex subset of $X$. In particular, if $A$ and $B$ are also finite dimensional, this shows $\operatorname{cocl}(A \cup B)$ (cocl denotes convex closure) is compact, convex, and finite dimensional. In our case let $V$ and $W$ be finite dimensional subspaces of $X$ such that range $(g) \subset V$ and range $(h) \subset W$ and define $C_{i}^{\prime \prime}=C_{i} \cap V$ and $D_{j}^{\prime \prime}=D_{j} \cap W$. Let $U$ be a finite dimensional subspace of $X$ such that range $(\theta) \subset U$ and define $E_{i, j}^{\prime}=E_{i, j} \cap U$. Now define $F^{\prime \prime}=\bigcup_{i=1}^{m} \bigcup_{j=1}^{n}\left[\operatorname{cocl}\left(C_{i}^{\prime} \cup C_{i}^{\prime \prime} \cup E_{i, j}^{\prime}\right) \cup \operatorname{cocl}\left(D_{j}^{\prime} \cup D_{j}^{\prime \prime} \cup E_{i, j}^{\prime}\right)\right]$. By our above remarks, $F^{\prime}$ is finite union of compact, convex, finite dimensional sets, and hence an ENR. It is also easy to see that $F^{\prime} \subset C$, $C^{\prime} \cup D^{\prime} \subset F^{\prime}$ and $\theta(G) \cup g(G) \cup h(G) \subset F^{\prime}$. We thus see that $i_{F^{\prime}}(g, G \cap$ $\left.F^{\prime \prime}\right), i_{F^{\prime}}\left(\theta, G \cap F^{\prime \prime}\right)$, and $i_{F^{\prime}}\left(h, G \cap F^{\prime}\right)$ are defined. Because $g\left(G \cap F^{\prime}\right) \subset C^{\prime}$ and $h\left(G \cap F^{\prime}\right) \subset D^{\prime}$, it follows (by the commutativity property) that $i_{C^{\prime}}\left(g, G \cap C^{\prime}\right)=i_{F^{\prime}}\left(g, G \cap F^{\prime}\right)$ and similarly for $h$. Thus to show that $i_{C^{\prime}}\left(g, G \cap C^{\prime}\right)=i_{D^{\prime}}\left(h, G \cap D^{\prime}\right)$, it suffices to show $i_{F^{\prime}}\left(g, G \cap F^{\prime}\right)=$ $i_{F^{\prime}}\left(\theta, G \cap F^{\prime \prime}\right)=i_{F^{\prime}}\left(h, G \cap F^{\prime \prime}\right)$. We prove that $i_{F^{\prime}}\left(g, G \cap F^{\prime \prime}\right)=i_{F^{\prime}}\left(\theta, G \cap F^{\prime \prime}\right)$, the proof for $h$ being the same. Consider the homotopy $s g(x)+$ $(1-s) \theta(x), 0 \leqq s \leqq 1, x \in \operatorname{cl}\left(G \cap F^{\prime \prime}\right)$. For $x \in \operatorname{cl}\left(G \cap F^{\prime \prime}\right)$, we know that $f(x) \in E_{i j}$ for some $i, j$, so $\theta(x) \in E_{i j}^{\prime}, g(x) \in C_{i}^{\prime \prime}$, and $s g(x)+(1-s) \theta(x) \in$ $\operatorname{cocl}\left[E_{i j}^{\prime} \cup C_{i}^{\prime \prime}\right] \subset F^{\prime \prime}$. Also, since $q_{1}(f(x)-s g(x)-(1-s) \theta(x)) \leqq s q_{1}(f(x)-$ $g(x))+(1-s) q_{1}(f(x)-\theta(x))<\varepsilon_{1} \quad$ and since $q_{1}(f(x)-x) \geqq \varepsilon_{1}$ for $x \in \partial G, s g(x)+(1-s) \theta(x) \neq x$ for $x \in \operatorname{cl}\left(G \cap F^{\prime \prime}\right)-G \cap F^{\prime}$. Thus the homotopy is permissible and $i_{F^{\prime}}\left(g, G \cap F^{\prime}\right)=i_{F^{\prime}}\left(\theta, G \cap F^{\prime \prime}\right)$.

Definition. Suppose that $C \in \mathscr{F}_{0}, C \subset X$ an lctvs, $G$ is an open 
subset of $C$, and $F: \operatorname{cl}(G) \rightarrow C$ is a continuous map such that $f(x) \neq x$ for $x \in \partial G$. Let $\left\{C_{i}: 1 \leqq i \leqq m\right\}$ be a covering of $C$ by compact, convex sets $C_{i} \subset C$ and let $g$ be an admissible approximation with respect to $\left\langle f,\left\{C_{i}\right\}\right\rangle$. Let $C_{i}^{\prime}, 1 \leqq i \leqq n$, be any finite dimensional compact convex sets such that $C_{i}^{\prime} \subset C_{i}$ and $g(G) \subset \bigcup_{i=1}^{m} C_{i}^{\prime} \equiv C^{\prime}$. Then we define $i_{C}(f, G)=i_{C^{\prime}}\left(g, G \cap C^{\prime}\right)$.

Theorem 1 shows that $g$ exists and that our definition does not depend on the particular admissible approximation $g$ or the particular $C_{i}^{\prime}$. Notice that if $C$ happens to be an ENR, so that $i_{C}(f, G)$ is already defined, then our definition reduces to the usual. To see this, just consider the homotopy $F(x, s)=(1-s) f(x)+s g(x), x \in \mathrm{cl}(G), 0 \leqq s \leqq 1$. Since $g$ is an admissible approximation, if $f(x) \in C_{i}$, then $g(x) \in C_{i}$, so that $F(x, s) \in C_{i}$. Furthermore, condition 1 on $g$ guarantees that $F(x, s) \neq x$ for $x \in G, 0 \leqq s \leqq 1$. It follows that $i_{C}(f, G)=i_{C}(g, G)$, and since $g(G) \subset C^{\prime}$, the commutativity property implies $i_{C}(g, G)=i_{C^{\prime}}\left(g, G \cap C^{\prime}\right)$.

It is now easy to show that the various theorems about the fixed point index for ENR's extend to our context.

THEOREM 2. Suppose $C \in \mathscr{F}_{0}, G$ is an open subset of $C$, and $f: \mathrm{cl}(G) \rightarrow C$ is a continuous map such that $f(x) \neq x$ for $x \in \partial G$. Then if $i_{C}(f, G) \neq 0, f$ has a fixed point in $G$. If $S=\{x \in G: f(x)=x\} \subset$ $G_{1} \cup G_{2}$, where $G_{1}$ and $G_{2}$ are disjoint open subsets of $G$, then $i_{C}(f, G)=$ $i_{C}\left(f, G_{1}\right)+i_{C}\left(f, G_{2}\right)$.

Proof. Suppose $i_{C}(f, G) \neq 0$. Since $C \in \mathscr{F}_{0}$, there exist compact, convex sets $C_{i}, 1 \leqq i \leqq n$, such that $C=\bigcup_{i=1}^{n} C_{i}$. By Theorem 1 , for any continuous seminorm $p$ and $\varepsilon>0$, there exists an admissible approximation $g$ with respect to $\left\langle f,\left\{C_{i}\right\}>\right.$ such that $p(g(x)-f(x))<\varepsilon$ for $x \in \mathrm{cl}(G)$; and furthemore $i_{C^{\prime}}\left(g, G \cap C^{\prime}\right)=i_{C}(f, G) \neq 0$, where $C^{\prime} \equiv$ $\bigcup_{i=1}^{n} C_{i}^{\prime}$ and $C_{i}^{\prime}$ are any compact, convex finite dimensional subsets of $C_{i}$, such that $g(G) \subset C^{\prime}$. By the usual additivity property there exists $x \in G \cap C^{\prime}$ such that $g(x)=x$, whence $p(f(x)-x)<\varepsilon$. It follows that if, for any continuous seminorm $p$ and $\varepsilon>0$, we define $C_{\varepsilon, p}=\{x \in$ $\operatorname{cl}(G): p(f(x)-x) \leqq \varepsilon\}, C_{\varepsilon, p}$ is a nonempty, compact set. Also, the collection $\left\{C_{\varepsilon, p}: p\right.$ a continuous, seminorm $\left.\varepsilon>0\right\}$ has the finite intersection property, since $\bigcap_{i=1}^{n} C_{\varepsilon_{i}, p_{i}} \subset C_{\varepsilon, q}$, where $\varepsilon=\min \left\{\varepsilon_{i}: 1 \leqq i \leqq n\right\}$ and $q(x)=\max \left\{p_{i}(x): 1 \leqq i \leqq n\right\}$. Thus there exists $x_{0} \in \cap C_{\varepsilon, p}$, and since $p\left(f\left(x_{0}\right)-x_{0}\right)=0$ for all continuous seminorms, $f\left(x_{0}\right)=x_{0}$.

To prove the second part of the theorem, note that $f(x) \neq x$ for $x \in \partial G \cup \partial G_{1} \cup \partial G_{2}$, so that exists a continuous seminorm $p$ and $\varepsilon>0$ such that $p(f(x)-x) \geqq \varepsilon$ for $x \in \partial G \cup \partial G_{1} \cup \partial G_{2}$. By Theorem 1, there exists an admissible approximation $g$ with respect to $<f,\left\{C_{i}\right\}>$ such 
that $p(f(x)-x)<\varepsilon$ for $x \in \operatorname{cl}(G)$, and we have $i_{C}(f, G)=i_{C^{\prime}}\left(g, G \cap C^{\prime}\right)$, $i_{C}\left(f, G_{i}\right)=i_{C^{\prime}}\left(g, G_{i} \cap C^{\prime}\right)$, as usual, $i=1,2$. By the additivity property for ENR's, $i_{C^{\prime}}\left(g, G \cap C^{\prime}\right)=i_{C^{\prime}}\left(g, G_{1} \cap C^{\prime}\right)+i_{C^{\prime}}\left(g, G_{2} \cap C^{\prime}\right)$.

With the aid of Theorem 2 a slight, but useful generalization of our previous definition can be given.

Definition. Suppose that $C \in \mathscr{F}_{0}, G$ is an open subset of $C$, and $f: G \rightarrow C$ is a continuous map such that $S=\{x \in G: f(x)=x\}$ is compact. Then we define $i_{c}(f, G)=i_{c}(f, V)$, where $V$ is any open neighborhood of $S$ such that $\mathrm{cl}(V) \subset G$.

This definition makes sense, for if $V_{1}$ and $V_{2}$ are two open neighborhoods of $S$ above such that $\mathrm{cl}\left(V_{i}\right) \subset G$, define $V=V_{1} \cap V_{2}$. Then if we set $U_{i}=V_{i}-\operatorname{cl}(V) i_{c}\left(f, V_{i}\right)-i_{c}\left(f, U_{i}\right)=i_{c}(f, V)$, by Theorem 2. Also we have $i_{C}\left(f, U_{i}\right)=0$, since $f$ has no fixed points in $U_{i}$ and Theorem 2 would imply $f$ had fixed points in $U_{i}$ if $i_{C}\left(f, U_{i}\right) \neq 0$. This shows $i_{c}\left(f, V_{1}\right)=i_{c}\left(f, V_{2}\right)$.

Henceforth we shall use this generalized definition. It is clear that Theorem 2 immediately generalizes to this context, the only difference in hypotheses being that we only assume $f$ is defined on $G$ and $S=\{x \in G: f(x)=x\}$ is compact.

Theorem 3. Suppose that $C \in \mathscr{F}_{0}, G$ is an open subset of $C, I=$ $[0,1]$, the closed unit interval, and $F: G \times I \rightarrow C$ is a continuous map such that $S=\{(x, t) \in G \times I: F(x, t)=x\}$ is compact. Then if we define $F_{t}: G \rightarrow C$ by $F_{t}(x)=F(x, t), i_{C}\left(F_{0}, G\right)=i_{C}\left(F_{1}, G\right)$.

Proof. Define $\pi: G \times I \rightarrow G$ by $\pi(x, t)=x$. It is clear that $\pi$ is a continuous map, so $\pi(S)=T$ is a compact subset of $G$. Let $V$ be an open neighborhood of $T$ such that $\operatorname{cl}(V) \subset G$. Since $\{F(x, t)-$ $x:(x, t) \in \partial V \times I\}$ is a compact set not containing 0 , there exists a continuous seminorm $p$ and $\varepsilon>0$ such that $p(F(x, t)-x) \geqq \varepsilon$ for $(x, t) \in$ $\partial V \times I$. Suppose $C=\bigcup_{i=1}^{n} C_{i}, C_{i}$ compact and convex. By Lemma 2 there exists a continuous, finite dimensional map $r: C \rightarrow C$ such that for all $x \in C, p(r(x)-x)<\varepsilon$ and for all $x \in C$ and $1 \leqq i \leqq n r(x) \in C_{i}$ if $x \in C_{i}$. We set $C^{\prime}=\mathrm{U}_{i=1}^{n} \operatorname{cocl} r\left(C_{i}\right)$, an ENR, and we define $H(x, t)=$ $r(F(x, t))$ for $x \in \mathrm{cl}(V), t \in I$. By definition we have $i_{C}\left(F_{t}, G\right)=i_{C}\left(F_{t}, V\right)$ and $i_{C}\left(F_{t}, V\right)=i_{C^{\prime}}\left(H_{t}, V \cap C^{\prime}\right)$, since $H_{t}$ is an admissible approximation with respect to $\left\langle F_{t},\left\{C_{i}\right\}\right\rangle$ and $H_{t}(G) \subset C^{\prime}$, where $C^{\prime}$ is of the required form. Now by the ordinary homotopy property for ENR's, $i_{C^{\prime}}\left(H_{0}, V ! \cap C^{\prime}\right)=i_{C^{\prime}}\left(H_{1}, V \cap C^{\prime}\right)$.

Corollary. Suppose that $C \in \mathscr{F}_{0}, G$ is an open subset of $C$, and $F: \operatorname{cl} G \times[0,1]$ is a continuous map such that $F(x, t) \neq x$ for $x \in \partial G$, 
$0 \leqq t \leqq 1$. Then $i_{C}\left(F_{1}, G\right)=i_{C}\left(F_{0}, G\right)$

Theorem 4. Suppose that $C \in \mathscr{F}_{0}$ and $f: C \rightarrow C$ is a continuous map. Then $H_{n}(C)$ (singular homology with rational coefficients) is a finite dimentional vector space for all $n$ and $H_{n}(C)=0$ except for finitely many $n$. Furthermore, if $\Lambda(f)=\sum_{n \geqq 0}(-1)^{n} \operatorname{tr}\left(f_{*}, n\right)$, the Lefschetz number of $f$, then $\Lambda(f)=i_{C}(f, C)$.

Proof. Since $C \in \mathscr{F}_{0}$, suppose $C=\bigcup_{i=1}^{n} C_{i}, C_{i}$ compact and convex. By Lemma 2, there exists a continuous finite dimensional map $r: C \rightarrow C$ such that $r(x) \in C_{i}$ if $x \in C_{i}$. As before we define $C^{\prime}=\bigcup_{i=1}^{n} \operatorname{cocl} r\left(C_{i}\right)$, an ENR. Recall that for any ENR $E$ (or in fact any compact, metric ANR [1]) $H_{n}(E)$ is a finite dimensional vector space and $H_{n}(E)=0$ except for finitely many $n$. In our case let us view $r$ as a map from $C$ to $C^{\prime}$ and let $i: C^{\prime} \rightarrow C$ be the inclusion map. Since $i r$ is homotopic to $I$, the identity on $C$, by the homotopy $s(i r)+(1-s) I, 0 \leqq s \leqq 1$, $(i r)_{*, n}=i_{*, n} r_{*, n}=I_{*, n}$. It follows that $i_{*, n}: H_{n}\left(C^{\prime}\right) \rightarrow H_{n}(C)$ must be onto, so $H_{n}(C)$ is a finite dimensional vector space and 0 for almost all $n$.

To show the second part of the theorem, note that irf is an admissible approximation to $\left\langle f,\left\{C_{i}\right\}>\right.$ so by definition we have $i_{C}(f, C)=$ $i_{C^{\prime}}\left(r f i, C^{\prime}\right)=$ (by the normalization property for ENR's) $\Lambda(r f i)$. Since $\operatorname{irf}$ is homotopic to $f$ (by the homotopy $s(\operatorname{irf})(x)+(1-s) f(x), 0 \leqq$ $s \leqq 1), \Lambda(f)=\Lambda(\operatorname{irf})$. However, we have $\left.\operatorname{tr}(\operatorname{irf})_{*, n}\right)=\operatorname{tr}\left(i_{*, n}(r f)_{*, n}\right)=$ $\operatorname{tr}\left((r f)_{*, n} i_{*, n}\right)=\operatorname{tr}\left((r f i)_{*, n}\right)$, by the commutativity property for the trace operator $t r$ on linear operators between finite dimensional vector spaces. This shows $\Lambda(r f i)=\Lambda(i r f)=\Lambda(f)$.

The proof of the commutativity property is a little more involved than that of Theorems 2-4. First, we need some simple lemmas.

Lemma 3. Let $K$ be a compact subset of an letvs $X$ and $f: K \rightarrow Y$ a continuous map of $K$ into an lctvs $Y$. Then given any continuous seminorm $q$ on $Y$ and $\varepsilon>0$, there exists a continuous seminorm $p$ on $X$ and $\delta>0$ such that for all $x, y \in K$ with $p(x-y)<\delta, q(f(x)-f(y))<\varepsilon$.

Proof. For each $x \in K$, there exists a seminorm $p_{x}$ and $\delta>0$ such that if $y \in K$ and $p_{x}(y-x)<\delta_{x}, q(f(y)-f(x))<\varepsilon / 2$. Let $N_{x}=$ $\left\{y \in K: p_{x}(y-x)<\delta_{x} / 2\right\}$ an open neighborhood of $x$ in $K$. The open sets $N_{x}$ give an open covering of $K$, and since $K$ is compact there exists a finite subcovering $N_{x_{1}}, \cdots, N_{x_{n}}$. Let $p(x)=\max _{1 \leqq i \leqq n}\left\{p_{x_{i}}(x)\right\}$ and $2 \delta=\min _{1 \leqq i \leqq n}\left\{\delta_{x_{i}}\right\}$; of course $p$ is a continuous seminorm. For convenience let $N_{x_{i}}=N_{i}, p_{x_{i}}=p_{i}$, and $\delta_{x_{i}}=\delta_{i}$. If we take $x, y \in K$ 
with $p(x-y)<\delta$, we can assume $x \in N_{i}$ for some $i$. Thus $p_{i}\left(x-x_{i}\right)<$ $\delta_{i} / 2$ and $p_{i}\left(y-x_{i}\right) \leqq p_{i}(y-x)+p_{i}\left(x-x_{i}\right) \leqq p(y-x)+p_{i}\left(x-x_{i}\right)<\delta_{i}$. It follows that $q\left(f(x)-f\left(x_{i}\right)\right)<\varepsilon / 2$ and $q\left(f(y)-f\left(x_{i}\right)\right)<\varepsilon / 2$, so that $q(f(y)-f(x))>\varepsilon$.

Lemma 4. Let $K$ be a compact subset of an letvs $X$. Let $G$ be an open subset of $K$. Then given any compact subset $V \subset G$, there exists a continuous seminorm $p$ and $\varepsilon>0$ such that $\{y \in K: x \in V$ with $p(y-x)<\varepsilon\} \equiv N_{p, \varepsilon}(V) \cap K \subset G$.

Proof. For each $x \in V$ there exists a continuous seminorm $p_{x}$ and $\varepsilon_{x}>0$ such that $\left\{y \in K: p_{x}(y-x)<\varepsilon_{x}\right\} \subset G$. Let $N_{x}=\left\{y \in K: p_{x}(y-x)<\right.$ $\left.\varepsilon_{x} / 2\right\}$. Since $K$ is compact there exists a finite subcovering $N_{x_{1}}, N_{x_{2}}, \cdots$, $N_{x_{n}}$ of the open covering $\left\{N_{x}\right\}$ of $V$. As before we define $p(x)=$ $\max _{1 \leqq i \leqq n}\left\{p_{x_{i}}(x)\right\}$ and $2 \varepsilon=\min _{1 \leqq i \leqq n}\left\{\varepsilon_{x_{i}}\right\}$. Then if $y \in N_{p, \varepsilon}(V) \cap K$, so that there exists $x \in V$ such that $p(y-x)<\varepsilon$, select $i$ such that $x \in N_{x_{i}}$. Then $p(y-x)<\varepsilon$ implies $p_{x_{i}}(y-x)<\varepsilon_{x_{i}} / 2$, and since $p\left(x-x_{i}\right)<\varepsilon_{x_{i}} / 2$, $p_{x_{i}}\left(y-x_{i}\right)<\varepsilon_{x i}$, whence $y \in G$.

THEOREM 5. (Commutativity property). Assume that $C_{k} \in \mathscr{F}_{0}$, $C_{k} \subset$ an letvs $X_{k}, k=1,2$. Let $G_{k} \subset C_{k}$ be open subsets of $C_{k}$ and let $f_{1}: G_{1} \rightarrow C_{2}$ and $f_{2}: G_{2} \rightarrow C_{1}$ be continuous maps. Define $H_{1}=f_{1}^{-1}\left(G_{2}\right)$ and $H_{2}=f_{2}^{-1}\left(G_{1}\right)$ and assume that $S_{1}=\left\{x \in H_{1}:\left(f_{2} f_{1}\right)(x)=x\right\}$ is compact. Then $S_{2}=\left\{x \in H_{2}:\left(f_{1} f_{2}\right)(x)=x\right\}$ is compact and $i_{C_{1}}\left(f_{2} f_{1}, H_{1}\right)=i_{C_{2}}\left(f_{1} f_{2}, H_{2}\right)$.

proof. The fact that $S_{2}$ is compact is immediate, since $f_{1}: S_{1} \rightarrow S_{2}$, $f_{2}: S_{2} \rightarrow S_{1},\left(f_{2} f_{1}\right)(x)=x$ for $x \in S_{1}$, and $\left(f_{1} f_{2}\right)(y)=y$ for $y \in S_{2}$.

Let $\widetilde{G}_{1}$ and $\widetilde{G}_{2}$ be open neighborhoods of $S_{1}$ and $S_{2}$ respectively such that $\operatorname{cl}\left(\widetilde{G}_{i}\right) \subset G_{i}$. Let $\widetilde{H}_{1}=\left\{x \in \widetilde{G}_{1}: f_{1}(x) \in \widetilde{G}_{2}\right\}$ and similarly for $H_{2}$. It is clear that $\widetilde{H}_{i}$ is an open neighborhood of $S_{i}$ and cl $\widetilde{H}_{i} \subset H_{i}$, so that $\left(f_{2} f_{1}\right)(x) \neq x$ for $x \in \operatorname{cl} \widetilde{H}_{1}-S_{1}$ and similarly $\left(f_{1} f_{2}\right)(x) \neq x$ for $x \in \operatorname{cl} \widetilde{H}_{2}-S_{2}$. By Theorem 2, $i_{C_{1}}\left(f_{2} f_{1}, \widetilde{H}_{1}\right)=i_{C_{1}}\left(f_{2} f_{1}, H_{1}\right)$ and similarly for $f_{1} f_{2}$. Thus we may as well assume at the start that $f_{i}$ is defined on cl $\left(G_{i}\right), S_{1}$ is a compact subset of $H_{1}=\left\{x \in G_{1}: f_{1}(x) \in G_{2}\right\}, S_{2}$ is a compact subset of $H_{2},\left(f_{2} f_{1}\right)(x) \neq x$, for $x \in \operatorname{cl} H_{1}-S_{1}$ and $\left(f_{1} f_{2}\right)(x) \neq x$ for $x \in \operatorname{cl~} H_{2}-S_{2}$. Let $U_{i}$ be a compact neighborhood of $S_{i}$ such that $U_{i} \subset H_{i}$ and let $V_{i}$ be an neighborhood of $S_{i}$ such that $\operatorname{cl} V_{i} \subset H_{i}$, $f_{1}\left(V_{1}\right) \subset U_{2}$ and $f_{2}\left(V_{2}\right) \subset U_{1}$. Theorem 2 implies $i_{C_{1}}\left(f_{2} f_{1}, H_{1}\right)=i_{C_{1}}\left(f_{2} f_{1}, V_{1}\right)$ and $i_{C_{2}}\left(f_{1} f_{2}, H_{2}\right)=i_{C_{2}}\left(f_{1} f_{2}, V_{2}\right)$, so it is enough to show that $i_{C_{1}}\left(f_{2} f_{1}, V_{1}\right)=$ $i_{C_{2}}\left(f_{1} f_{2}, V_{2}\right)$.

Since $\left(f_{2} f_{1}\right)(x)-x \neq 0$ for $x \in \operatorname{cl}\left(H_{1}\right)-V_{1}$ (a compact set), there exists a continuous seminorm $p_{1}$ on $X_{1}$ and $\varepsilon_{1}>0$ such that $p_{1}\left(\left(f_{2} f_{1}\right)(x)-\right.$ $x) \geqq \varepsilon_{1}$ for $x \in \operatorname{cl}\left(H_{1}\right)-V_{1}$. Similarly, there exists a continuous semi- 
norm $p_{2}$ on $X_{2}$ and $\varepsilon_{2}>0$ such that $p_{2}\left(\left(f_{1} f_{2}\right)(x)-x\right)>\varepsilon_{2}$ for $x \in \operatorname{cl}\left(H_{2}\right)-V_{2}$. By Lemmas 3 and 4 there exist a continuous seminorm $q_{1}$ on $X_{1}$ and $\delta_{1}>0$ such that $\left\{y \in C_{1}: x \in U_{1}\right.$ with $\left.q_{1}(y-x)<\delta_{1}\right\} \equiv N_{q_{1}, \delta_{1}}\left(U_{1}\right) \cap C_{1} \subset H_{1}$ and for all $y, z \in \mathrm{cl}\left(G_{1}\right)$ such that $q_{1}(y-z)<\delta_{1}, p_{2}\left(f_{1}(y)-f_{1}(z)\right)<\varepsilon_{2} / 2_{2}$. For the same reasons there exist a continuous seminorm $q_{2}$ on $X_{2}$ and $\delta_{2}>0$ such that $N_{q_{2}, \delta_{2}}\left(U_{2}\right) \cap C_{2} \subset H_{2}$ and for all $y, z \in \operatorname{cl}\left(G_{2}\right)$ such that $q_{2}(y-z)<\delta_{2}, p_{1}\left(f_{2}(y)-f_{2}(z)\right)<\varepsilon_{1} / 2$. Because $C_{1}, C_{2} \in \mathscr{F}_{0}, C_{1}=\bigcup_{i=1}^{m} C_{1, i}$ and $C_{2}=$ $\mathrm{U}_{j=1}^{n} C_{2, j}, C_{1, j}$ and $C_{2, j}$ compact, convex sets. By Lemma 2 there exists a continuous, finite dimensional map $r_{1}: C_{1} \rightarrow C_{1}$ such that for all $x \in C_{1}, r_{1}(x) \in C_{1 i}$ if $x \in C_{1, i}, p_{1}\left(r_{1}(x)-x\right)<\varepsilon_{1} / 2$, and $q_{1}\left(r_{1}(x)-x\right)<\delta_{1}$. For the same reason there exists a continuous finite dimensional map $r_{2}: C_{2} \rightarrow C_{2}$ such that for all $x \in C_{2}, r_{2}(x) \in C_{2, j}$ if $x \in C_{2, j}, p_{2}\left(r_{2}(x)-x\right)<\varepsilon_{2} / 2$ and $q_{2}\left(r_{2}(x)-x\right)<\delta_{2}$. As usual we define $C_{1}^{\prime}=\bigcup_{i=1}^{m} \operatorname{cocl} r_{1}\left(C_{1, i}\right)$ and $C_{2}^{\prime}=\bigcup_{j=1}^{n} \operatorname{cocl} r_{2}\left(C_{2, j}\right)$.

We now define two supplementary functions, $g_{1}=r_{2} f_{1} \mid H_{1}$ and $g_{2}=r_{1} f_{2} \mid H_{2}$. Since $q_{2}\left(r_{2} f_{1}(x)-f_{1}(x)\right)<\delta_{2}, f_{1}(x) \in U_{2}$ for $x \in \operatorname{cl} V_{1}$, and $N_{q_{2} \cdot \delta_{2}}\left(U_{2}\right) \cap C_{2} \subset H_{2}$, we see that $g_{1}\left(\mathrm{cl} V_{1}\right) \subset H_{2}$. For the same reasons we observe that $g_{2}\left(\operatorname{cl} V_{2}\right) \subset H_{1}$. If we set $0_{1}=g_{1}^{-1}\left(H_{2}\right)$ and $0_{2}=g_{2}^{-1}\left(H_{1}\right)$, the above observations show that $\mathrm{cl} V_{1} \subset 0_{1}$ and $\mathrm{cl} V_{2} \subset 0_{2}$. We claim that $\left(g_{2} g_{1}\right)(x) \neq x$ for $x \in 0_{1}-V_{1}$ and $\left(g_{1} g_{2}\right)(x) \neq x$ for $x \in 0_{2}-V_{2}$. To prove this for $g_{2} g_{1}$, recall that for all $x \in 0_{1}$ (so $r_{2} f_{1}(x) \in H_{2}$ and $f_{1}(x) \in G_{1}$ ), $q_{2}\left(r_{2} f_{1}(x)-f_{1}(x)\right)<\delta_{2}$. By the assumption on $\delta_{2}$ this implies $p_{1}\left(f_{2} r_{2} f_{2}(x)-\right.$ $\left.f_{2} f_{1}(x)\right)<\varepsilon_{1} / 2$, while the assumption on $r_{1}$ guarantees that $p_{1}\left(r_{1} f_{2} r_{2} f_{1}(x)-\right.$ $\left.f_{2} r_{2} f_{1}(x)\right)<\varepsilon_{1} / 2_{2}$. Thus we see that for $x \in 0_{1}, p_{1}\left(g_{2} g_{1}(x)-f_{2} f_{1}(x)\right)<\varepsilon_{1}$, and since $p_{1}\left(f_{2} f_{1}(x)-x\right) \geqq \varepsilon_{1}$ for $x \in$ cl $H_{1}-V_{1}$, it follows that $g_{2} g_{1}(x) \neq x$ for $x \in 0_{1}-V_{1}$. The proof for $g_{1} g_{2}$ is the same. This observation shows that $i_{C_{1}}\left(g_{2} g_{1}, 0_{1}\right)$ and $i_{C_{1}}\left(g_{2} g_{1}, V_{1}\right)$ are defined and equal and similarly for $g_{1} g_{2}$.

Our next claim is that $i_{C_{1}}\left(g_{2} g_{1}, V_{1}\right)=i_{C_{1}}\left(f_{2} f_{1}, V_{1}\right)$. To see this we consider the homotopy $F$ : cl $V_{1} \times I \rightarrow C_{1}$ defined by $F(x, t)=(1-t) r_{1} f_{2}((1-$ t) $\left.r_{2} f_{1}(x)+t f_{1}(x)\right)+t f_{2}\left((1-t) r_{2} f_{1}(x)+t f_{1}(x)\right)$ and we apply Theorem 3 . We have to show that this homotopy is permissible. First note that for all $x \in \mathrm{cl}\left(V_{1}\right), f_{1}(x) \in U_{2}$ and $q_{2}\left(r_{2} f_{1}(x)-f_{1}(x)\right)<\delta_{2}$. It follows that $(1-t) r_{2} f_{1}(x)+t f_{1}(x) \in N_{q_{2}, \delta_{2}}\left(U_{2}\right) \cap C_{2} \subset H_{2}$. $\quad$ (Of course $(1-t) r_{2} f_{1}(x)+$ $t f_{1}(x) \in C_{2}$, since if $f_{1}(x) \in C_{2, j}, r_{2} f_{1}(x) \in C_{2, j}$ and hence $(1-t) r_{2} f_{1}(x)+$ $t f_{1}(x) \in C_{2 . j}$ for $\left.0 \leqq t \leqq 1\right)$. This shows that $f_{2}\left((1-t) r_{2} f_{1}(x)+t f_{1}(x)\right)$ is defined for $x \in \operatorname{cl} V_{1}$ and applying the usual reasoning we see that $F(x, t) \in C_{1}$ for $x \in \operatorname{cl} V_{1}, 0 \leqq t \leqq 1$. It remains to show that $F(x, t) \neq x$ for $x \in \partial V_{1}, 0 \leqq t \leqq 1$. We have seen above that $q_{2}\left(r_{2} f_{1}(x)-f_{1}(x)\right)<\delta_{2}$ and it follows that $\left.q_{2}\left((1-t) r_{2} f_{1}(x)+t f_{1}(x)\right)-f_{1}(x)\right)<(1-t) \delta_{2} \leqq \delta_{2}$. It follows that $p_{1}\left(f_{2}\left((1-t) r_{2} f_{1}(x)+t f_{1}(x)\right)-f_{2} f_{1}(x)\right)<\varepsilon_{1} / 2$ and since $p_{1}\left(r_{1}(y)-y\right)<\varepsilon_{1} / 2$ for $y \in C_{1}$ we conclude that $p_{1}\left(F(x, t)-f_{2} f_{1}(x)\right)<$ $(1-t) \varepsilon_{1}+t \varepsilon_{1} / 2$ or $p_{1}\left(F(x, t)-f_{2} f_{1}(x)\right)<\varepsilon_{1} \quad$ Since $p_{1}\left(f_{2} f_{1}(x)-x\right) \geqq \varepsilon$ for $x \in \partial V_{1}$, our homotopy is permissible and $i_{C_{1}}\left(f_{2} f_{1}, V_{1}\right)=i_{C_{1}}\left(g_{2} g_{1}, V_{1}\right)$. By 
the same reasoning we also find $i_{C_{2}}\left(f_{1} f_{2}, V_{2}\right)=i_{C_{2}}\left(g_{1} g_{2}, V_{2}\right)$.

To complete our proof it only remains to show that $i_{C_{2}}\left(g_{2} g_{1}, V_{1}\right)=$ $i_{C_{2}}\left(g_{1} g_{2}, V_{2}\right)$. However notice that $g_{2} g_{1}$ is an admissible approximation with respect to $\left\langle g_{2} g_{1},\left\{C_{1, i}\right\}>\right.$ and $g_{2} g_{1}\left(V_{1}\right) \subset C_{1}^{\prime}$, where $C_{1}^{\prime}$ is of the required form. By our definition it follows that $i_{C_{1}}\left(g_{2} g_{1}, V_{1}\right)=i_{C_{1}^{\prime}}\left(g_{2} g_{1}, V_{1} \cap\right.$ $\left.C_{1}^{\prime}\right)$. By the same reasoning we also see that $i_{C_{2}}\left(g_{1} g_{2}, V_{2}\right)=i_{C_{2}^{\prime}}\left(g_{1} g_{2}, V_{2} \cap\right.$ $\left.C_{2}^{\prime}\right)$. If we consider $h_{1}=g_{1} \mid C_{1}^{\prime} \cap H_{1}$ and $h_{2}=g_{2} \mid C_{2}^{\prime} \cap H_{2}$, it is easy to see that $h_{1}: C_{1}^{\prime} \cap H_{1} \rightarrow C_{2}^{\prime}, h_{2}: C_{2}^{\prime} \cap H_{2} \rightarrow C_{1}^{\prime}, h_{1}^{-1}\left(C_{2}^{\prime} \cap H_{2}\right)=0_{1} \cap C_{1}^{\prime}$ and $h_{2}^{-1}\left(C_{1}^{\prime} \cap H_{1}\right)=0_{1} \cap C_{2}^{\prime}$. Since we have already shown that $g_{2} g_{1}(x) \neq x$ for $x \in 0_{1}-V_{1}$ and cl $V_{1} \subset 0_{1}$, we thus see that $i_{C_{1}^{\prime}}\left(h_{2} h_{1}, h_{1}^{-1}\left(H_{2} \cap C_{2}^{\prime}\right)\right)$ is defined and equals $i_{C_{1}^{\prime}}\left(h_{2} h_{1}, V_{1} \cap C_{1}^{\prime}\right)$. For the same reasons we see that $i_{C_{2}^{\prime}}\left(h_{1} h_{2}, h_{2}^{-1}\left(H_{1} \cap C_{1}^{\prime}\right)\right)=i_{C_{2}^{\prime}}\left(h_{1} h_{2}, V_{2} \cap C_{2}^{\prime}\right)$. Since the ordinary commutativity property implies $i_{C_{1}^{\prime}}\left(h_{2} h_{2}, h_{1}^{-1}\left(H_{2} \cap C_{2}^{\prime}\right)\right)=i_{C_{2}^{\prime}}\left(h_{1} h_{2}, h_{2}^{-1}\left(H_{1} \cap C_{1}^{\prime}\right)\right)$, we are done.

2. In this section we shall define a fixed point index for continuous maps defined in topological spaces which are homeomorphic to retracts of spaces $C \in \mathscr{F}_{0}$. The method we shall use is not new, and we include this treatment for the sake of completeness. The basic technique of this section seems first to have been explicitly stated in its essentials by A. Deleanu [5]. A number of other authors, among them Dold [6], Browder [4], Granas [9] and Nussbaum [16] have also used variants of the same idea.

We begin with some notation. If $D$ is a compact, Hausdorff space, we write $D \in \mathscr{F}$ if there exists $C \in \mathscr{F}_{0}$, a continuous map $j: D \rightarrow C$, and a continuous map $r: C \rightarrow D$ such that $r j=I_{D}$, the identity on $D$. If $G$ is an open subset of $D$ and $f: G \rightarrow D$ is a continuous map such that $S=\{x \in G: f(x)=x\}$ is compact, it is clear that $T \equiv\left\{x \in r^{-1}(G):(j f r)(x)=\right.$ $x\} \subset r^{-1}(S)$, and since $r^{-1}(S)$ is a compact subset of $r^{-1}(G)$, it follows that $T$ is a compact subset of $r^{-1}(G)$. Thus $i_{C}\left(j f r, r^{-1}(G)\right)$ is defined. If we write $j f r=(j f)(r)$ and formally try to apply the commutativity property to $r: C \rightarrow D$ and $j f: G \rightarrow C$, we find that $i_{C}\left(j f r, r^{-1}(G)\right)=$ $i_{D}(r j f, G)=i_{D}(f, G)$. Thus it is natural to try to define $i_{D}(f, G)=$ $i_{c}\left(j f r, r^{-1}(G)\right)$. Our first theorem shows that this definition is welldefined.

Theorem 6. Suppose that $D \in \mathscr{F}$ and for $k=1,2$ suppose that $C_{k} \in \mathscr{F}_{0}$ and $j_{k}: D \rightarrow C_{k}$ and $r_{k}: C_{k} \rightarrow D$ are continuous maps such that $r_{k} j_{k}=I_{D}$, the identity on $D$. Let $G$ be an open subset of $D$ and $f: G \rightarrow D$ a continuous map such that $S=\{x \in G: f(x)=x\}$ is compact. Then $i_{C_{1}}\left(j_{1} f r_{1}, r_{1}^{-1}(G)\right)=i_{C_{2}}\left(j_{2} f r_{2}, r_{2}^{-1}(G)\right)$.

Proof. Write $j_{2} f r_{2}=\left(j_{2} r_{1}\right)\left(j_{1} f r_{2}\right)$ and define $h_{1}=j_{2} r_{1}: C_{1} \rightarrow C_{2}$ and 
$h_{2}=j_{1} f r_{2}: r_{2}^{-1}(G) \rightarrow C_{1}$. It is easy to check that $h_{2}^{-1}\left(C_{1}\right)=r_{2}^{-1}(G)$ and $h_{1}^{-1}\left(r_{2}^{-1}(G)\right)=r_{1}^{-1}(G)$, so it follows by Theorem 5 that $i_{C_{1}}\left(h_{2} h_{1}, r_{1}^{-1}(G)\right)=$ $i_{C_{2}}\left(h_{1} h_{2}, r_{2}^{-1}(G)\right)$. However $h_{2} h_{1}=j_{1} f r_{2} j_{2} r_{1}=j_{1} f r_{1}$, so we have the desired result.

Thus we can define $i_{D}(f, G)=i_{c}\left(j f r, r^{-1}(G)\right.$ ) (same notation as before). As immediate consequences of Theorems 2 and 3 we obtain the following theorems, whose proofs we omit.

THEOREM 7. Suppose that $D \in \mathscr{F}, G$ is an open subset of $D$, and $f: G \rightarrow D$ is a continuous map such that $S=\{x \in G: f(x)=x\}$ is compact. If $i_{D}(f, G) \neq 0$, then $f$ has a fixed point in $G$. If $S \subset G_{1} \cup G_{2}$, where $G_{1}$ and $G_{2}$ are disjoint open subsets of $G$, then $i_{C}(f, G)=i_{C}\left(f, G_{1}\right)+$ $i_{c}\left(f, G_{2}\right)$

THEOREM 8. Suppose that $D \in \mathscr{F}, G$ is an open subset of $D, I=$ $[0,1]$, and $F: G \times I \rightarrow D$ is a continuous map such that $S=\{(x, t) \in$ $G \times I: F(x, t)=x\}$ is compact. Then $i_{D}\left(F_{0}, G\right)=i_{D}\left(F_{1}, G\right)$.

Theorem 9. Suppose that $D \in \mathscr{F}$ and $f: D \rightarrow D$ is a continuous map. Then $\Lambda(f)$, the Lefschetz number of $f$, (singular homology with rational coefficients) is defined and $\Lambda(f)=i_{D}(f, D)$.

Proof. By definition there exist $C \in \mathscr{F}_{0}$ and continuous maps $j: D \rightarrow C$ and $r: C \rightarrow D$ such that $r j=I_{D}$, the identity on $D$. Since $r j=I_{D}, r_{*: n}: H_{n}(C) \rightarrow H_{n}(D)$ is onto and $H_{n}(D)$ is a finite dimensional vector space and 0 for almost all $n$. Again by definition $i_{D}(f, D)=$ $i_{c}(j f r, C)$; and since $i_{c}(j f r, C)=\Lambda(j f r)$, it suffices to show $\Lambda(f)=$ $\Lambda(j f r)$. However we have $\Lambda(j f r)=\sum_{n \geq 0}(-1)^{n} \operatorname{tr}\left((j f)_{*, n} r_{*, n}\right)=($ by the properties of trace) $\sum_{n \geqq 0}(-1)^{n} \operatorname{tr}\left(r_{*, n}(j f)_{*, n}\right)=\sum_{n \geqq 0}(-1)^{n} \operatorname{tr} f_{* n}=\Lambda(f)$.

THeorem 10. Assume that $D_{1}, D_{2} \in \mathscr{F}, G_{1}$ and $G_{2}$ are open subsets of $D_{1}$ and $D_{2}$ respectively, $f_{1}: G_{1} \rightarrow D_{2}$ and $f_{2}: G_{2} \rightarrow D_{1}$ are continuous maps. Let $H_{1}=f_{1}^{-1}\left(G_{2}\right), H_{2}=f_{2}^{-1}\left(G_{1}\right)$, and assume that $S_{1}=\left\{x \in H_{1}:\left(f_{2} f_{1}\right)(x)=x\right\}$ is compact. Then $S_{2}=\left\{x \in H_{2}:\left(f_{1} f_{2}\right)(x)=x\right\}$ is compact and $i_{D_{1}}\left(f_{2} f_{1}, H_{1}\right)=$ $i_{D_{2}}\left(f_{1} f_{2}, H_{2}\right)$.

Proof. The same proof as before shows $S_{2}$ is compact. Since $D_{k} \in \mathscr{F}, k=1,2$, there exist $C_{k} \in \mathscr{F}_{0}$ and continuous maps $j_{k}: D_{k} \rightarrow C_{k}$ and $r_{k}: C_{k} \rightarrow{ }_{k}$ such that $r_{k} j_{k}=I_{D_{k}}, k=1,2$. We have to show that $i_{C_{1}}\left(j_{1} f_{2} f_{1} r_{1}, r_{1}^{-1}\left(H_{1}\right)\right)=i_{C_{2}}\left(j_{2} f_{1} f_{2} r_{2}, r_{2}^{-1}\left(H_{2}\right)\right)$. Define $g_{1}=j_{2} f_{1} r_{1}: r_{1}^{-1}\left(G_{1}\right) \rightarrow C_{2}$ and $g_{2}=j_{1} f_{2} r_{2}: r_{2}^{-1}\left(G_{2}\right) \rightarrow C_{1}$. It is easy to check that $g_{1}^{-1}\left(r_{2}^{-1}\left(G_{2}\right)\right)=$ $r_{1}^{-1}\left(H_{1}\right)$ and $g_{2}^{-1}\left(r_{1}^{-1}\left(G_{1}\right)\right)=r_{2}^{-1}\left(H_{2}\right)$; also we see that $g_{2} g_{1}=j_{1} f_{2} f_{1} r_{1}$ and 
$g_{1} g_{2}=j_{2} f_{1} f_{2} r_{2}$. It follows by Theorem 5 applied to $g_{1}$ and $g_{2}$ that $i_{C_{1}}\left(j_{1} f_{2} f_{1} r_{1}, r_{1}^{-1}\left(H_{1}\right)\right)=i_{C_{2}}\left(j_{2} f_{1} f_{2} r_{2}, r_{2}^{-1}\left(H_{2}\right)\right)$.

REMARK. The method of proof we have used shows that there is a unique integer-valued function $i_{D}(f, G)$ (defined for $D \in \mathscr{F}, G$ an open subset of $D$, and $f: G \rightarrow D$ a continuons map such that $\{x \in G: f(x)=$ $x\}$ is compact) which satisfies Theorems 7-10. For as we have already remarked there is a unique such function defined for ENR's $D$. The methods of $\S 1$, using Theorem 1, the homotopy property, and the commutativity property, show that the index function is determined by its value for ENR's when $D \in \mathscr{F}_{0}$. Finally, we saw in this section that the commutativity property completely determined our definition in terms of the index for $D \in \mathscr{F}_{0}$.

\section{REFERENCES}

1. K. Borsuk, The Theory of Retracts.

2. F. E. Browder, The Topological Fixed Point Theory and its Applications in Functional Analysis, Princeton University, Ph. D. thesis, 1948.

3. - On the fixed point index for continuous mappings of locally connected spaces, Summa Brasiliensis Mathematicae 4 fasc., 7 (1960), 253-293.

4. - Nonlinear operators and nonlinear equations of evolution in Banach spaces, Proceedings of the Symposium on Nonlinear Functional Analysis, Amer. Math. Soc., April, 1968 (to appear).

5. A. Deleanu, Theorie des points fixes sur les retracter de voisinage des espaces convexoides, Bull. Soc. Math. France, 87 (1959), 235-243.

6. A Dold, Fixed point index and fixed point theorem for Euclidean neighborhood retracts, Topology, 4 (1965), 1-8.

7. J. Dugundji, An extension of Tietze's theorem, Pacific J. Math., 1 (1951), 353-367.

8. E. Dyer, $A$ fixed point theorem, Proc. Amer. Math. Soc., 7 (1956), 662-672.

9. A. Granas, Some theorems in fixed point theory. The Leray-Schauder index and the Lefschetz number, Bull. Polish Acad. Sci., 17 (1969), 181-138.

10. O. Hanner, Some theorems on absolute neighborhood retracts, Ark. Mat., 1 (1951), 398-408.

11. S. Lefschetz, Topics in Topology, Princeton University Press, Princeton, New Jersey, 1942.

12. J. Leray, Sur la forme des espaces topologiques et sur les points fixes des representations, J. de Math. Pures et Appl., 24 (1945).

13. - Sur la position d'un ensemble ferme de points d'un espace topologique, J. de Math. Pures et Appl., 24 (1945), 1969-199.

14. - Sur les equations et les transformations, J. de Math. Pures et Appl., 24 (1945), 201-248.

15. Theorie des points fixes, Indice total et nombre de Lefschetz, Bull. Soc. Math. France, 87 (1959), 221-233.

16. R. Nussbaum, The Fixed Point Index and Fixed Point Theorems for k-Set Contractions, University of Chicago Ph. D. Tndsis, 1969.

17. B. O'Neill, Essential sets and fixed points, Amer. J. Math., 75 (1953), 497-509.

18. J. Segal, $A$ fixed point theorem for the hyperspace of a snake-like continuum, Fund. Math., 50 (1962), 237-248. 
19. R. Thompson, A unified approach to local and global fixed point indices, Advances in Math., 3 (1969), 1-72.

Received May 4, 1970 and in revised form November 15, 1970.

RUtgers UNIVERSITY 


\title{
PACIFIC JOURNAL OF MATHEMATICS
}

\author{
EDITORS
}

\author{
H. SAMELSON \\ Stanford University \\ Stanford, California 94305 \\ C. R. HOBBY \\ University of Washington \\ Seattle, Washington 98105
}

J. DugundJI

Department of Mathematics

University of Southern California

Los Angeles, California 90007

RICHARD ARENS

University of California

Los Angeles, California 90024

\section{ASSOCIATE EDITORS}
E. F. BeCKenBaCH
B. H. NeUMANN
F. WOLF
K. Yoshida

\section{SUPPORTING INSTITUTIONS}

\author{
UNIVERSITY OF BRITISH COLUMBIA \\ CALIFORNIA INSTITUTE OF TECHNOLOGY \\ UNIVERSITY OF CALIFORNIA \\ MONTANA STATE UNIVERSITY \\ UNIVERSITY OF NEVADA \\ NEW MEXICO STATE UNIVERSITY \\ OREGON STATE UNIVERSITY \\ UNIVERSITY OF OREGON \\ OSAKA UNIVERSITY
}

\author{
UNIVERSITY OF SOUTHERN CALIFORNIA \\ STANFORD UNIVERSITY \\ UNIVERSITY OF TOKYO \\ UNIVERSITY OF UTAH \\ WASHINGTON STATE UNIVERSITY \\ UNIVERSITY OF WASHINGTON \\ AMERICAN MATHEMATICAL SOCIETY \\ NAVAL WEAPONS CENTER
}

The Supporting Institutions listed above contribute to the cost of publication of this Journal, but they are not owners or publishers and have no responsibility for its content or policies.

Mathematical papers intended for publication in the Pacific Journal of Mathematics should be in typed form or offset-reproduced, (not dittoed), double spaced with large margins. Underline Greek letters in red, German in green, and script in blue. The first paragraph or two must be capable of being used separately as a synopsis of the entire paper. The editorial "we" must not be used in the synopsis, and items of the bibliography should not be cited there unless absolutely necessary, in which case they must be identified by author and Journal, rather than by item number. Manuscripts, in duplicate if possible, may be sent to any one of the four editors. Please classify according to the scheme of Math. Rev. Index to Vol. 39. All other communications to the editors should be addressed to the managing editor, Richard Arens, University of California, Los Angeles, California, 90024.

50 reprints are provided free for each article; additional copies may be obtained at cost in multiples of 50 .

The Pacific Journal of Mathematics is published monthly. Effective with Volume 16 the price per volume (3 numbers) is $\$ 8.00$; single issues, $\$ 3.00$. Special price for current issues to individual faculty members of supporting institutions and to individual members of the American Mathematical Society: $\$ 4.00$ per volume; single issues $\$ 1.50$. Back numbers are available.

Subscriptions, orders for back numbers, and changes of address should be sent to Pacific Journal of Mathematics, 103 Highland Boulevard, Berkeley, California, 94708.

PUBLISHED BY PACIFIC JOURNAL OF MATHEMATICS, A NON-PROFIT CORPORATION

Printed at Kokusai Bunken Insatsusha (International Academic Printing Co., Ltd.), 270, 3chome Totsuka-cho, Shinjuku-ku, Tokyo 160, Japan. 


\section{Pacific Journal of Mathematics}

\section{Vol. 39, No. $3 \quad$ July, 1971}

William O'Bannon Alltop, 5-designs in affine spaces ................... 547

B. G. Basmaji, Real-valued characters of metacyclic groups ................. 553

Miroslav Benda, On saturated reduced products....................... 557

J. T. Borrego, Haskell Cohen and Esmond Ernest Devun, Uniquely representable semigroups. II.......................................

George Lee Cain Jr. and Mohammed Zuhair Zaki Nashed, Fixed points and stability for a sum of two operators in locally convex spaces ....................

Donald Richard Chalice, Restrictions of Banach function spaces ...............

Eugene Frank Cornelius, Jr., A generalization of separable groups ..............

Joel L. Cunningham, Primes in products of rings ......................

Robert Alan Morris, On the Brauer group of $Z$.

593

603

615

David Earl Dobbs, Amitsur cohomology of algebraic number rings ...............

Charles F. Dunkl and Donald Edward Ramirez, Fourier-Stieltjes transforms and

weakly almost periodic functionals for compact groups ...................

Hicham Fakhoury, Structures uniformes faibles sur une classe de cônes et

d'ensembles convexes ......................................

Leslie R. Fletcher, A note on C $\theta \theta$-groups.

Humphrey Sek-Ching Fong and Louis Sucheston, On the ratio ergodic theorem for

semi-groups............................................

James Arthur Gerhard, Subdirectly irreducible idempotent semigroups ...........

Thomas Eric Hall, Orthodox semigroups.....................

Marcel Herzog, $C \theta \theta$-groups involving no Suzuki groups ..........

669

687

John Walter Hinrichsen, Concerning web-like continua ..........

691

Frank Norris Huggins, A generalization of a theorem of F. Riesz.

695

Carlos Johnson, Jr., On certain poset and semilattice homomorphisms

703

Alan Leslie Lambert, Strictly cyclic operator algebras ...........

717

Howard Wilson Lambert, Planar surfaces in knot manifolds . . .

727

Robert Allen McCoy, Groups of homeomorphisms of normed linear spaces ....... 735

T. S. Nanjundiah, Refinements of Wallis's estimate and their generalizations ...... 745

Roger David Nussbaum, A geometric approach to the fixed point index .......... 751

John Emanuel de Pillis, Convexity properties of a generalized numerical range .... 767

Donald C. Ramsey, Generating monomials for finite semigroups ....

783

William T. Reid, A disconjugacy criterion for higher order linear vector differential equations...

Roger Allen Wiegand, Modules over universal regular rings...

Kung-Wei Yang, Compact functors in categories of non-archimedean Banach

spaces.

R. Grant Woods, Correction to: "Co-absolutes of remainders of Stone-Čech compactifications".

Ronald Owen Fulp, Correction to: "Tensor and torsion products of

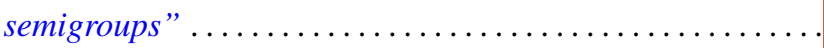

Bruce Alan Barnes, Correction to: "Banach algebras which are ideals in a banach 